\title{
Influencia de los factores psicosociales en adolescentes con Diabetes Mellitus tipo I $\left(^{*}\right)$
}

Influence of psychosocial factors in adolescents suffering from Diabetes Mellitus type $I\left({ }^{\star}\right)$

\section{Influência de fatores psicossociais em adolescentes que sofrem de Diabetes Mellitus tipo I(*)}

\author{
María Cristina Real Pérez ${ }^{1}$, Sergio Romeo López Alonso ${ }^{1,2}$ \\ ${ }^{1}$ Centro Universitario de Enfermería "San Juan de Dios”. Bormujos, Sevilla (España). \\ ${ }^{2}$ Dispositivo de Cuidados Críticos y Urgencias. Distrito Sanitario Málaga. Servicio Andaluz de Salud. Málaga (España). \\ ${ }^{*}$ ) El contenido de este artículo pertenece al Trabajo de Fin de Grado presentado en el Centro Universitario de Enfermería \\ "San Juan de Dios" durante el año académico 2014-2015. \\ Agradecimientos: Gracias a los profesionales del C.U.E. San Juan de Dios de Bormujos por la colaboración y el apoyo en la \\ finalización de este trabajo, en especial a los enfermeros y enfermeras del centro. \\ Cómo citar este artículo en edición digital: Real Pérez, Ma C., \& López Alonso, S.R. (2017). Influencia de los factores psicoso- \\ ciales en adolescentes con Diabetes Mellitus tipo I. Cultura de los Cuidados (Edición digital), 21(48). Recuperado de < http:// \\ dx.doi.org/10.14198/cuid.2017.48.21> \\ Correspondencia: Ma Cristina Real Pérez. Hospital San Juan de Dios del Aljarafe, en la dirección Avda. \\ San Juan de Dios s/n CP 41930, Bormujos (Sevilla). \\ Correo electrónico: cristinareal93@gmail.com \\ Recibido: 19/12/2016; Aceptado: 07/03/2017
}

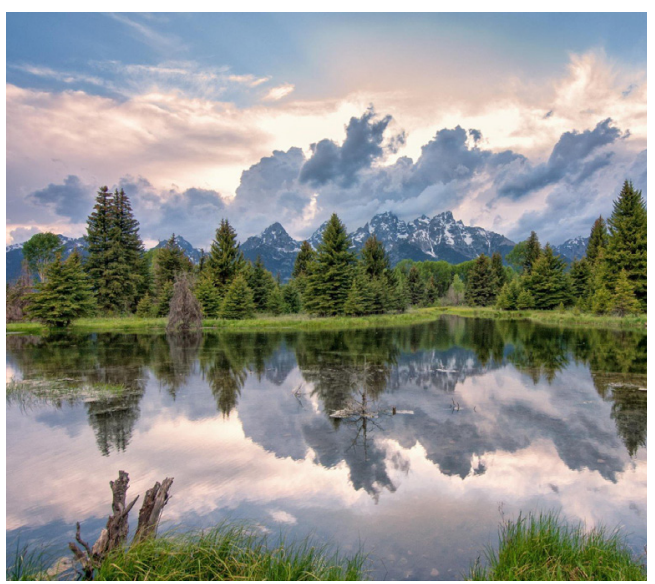

\section{ABSTRACT}

During adolescence many psychosocial factors that can alter the development process to adulthood are presented. In addition adolescents with diabetes mellitus type I, this development is far more complicated, causing impairment of the proper maintenance of this chronic disease.
Objective: To know the influence of psychosocial factors on the control of Diabetes Mellitus in adolescence.

Metholodology: One literature review was performed on PubMed, CINAHL, Scielo, CUIDEN, Spanish Medical Index, Dialnet, in addition to Google Scholar data and other specialized organizations website, selecting research articles of the last 10 years as the primary inclusion.

Results-Discussion: As results are eight main factors that influence the control of diabetes in adolescents, they are family, friends, anxiety and depression, diet and nutrition, stress, self-esteem, cognitive development and culture and religion .

Conclusion: Must be taken into account not only the physical and biological factors, but all the psychological and social aspects that also surround the teenager. Nurses should be aware of these factors when dealing with 
adolescents, since they can be the cause of your imbalance of blood glucose, so it is always necessary to assess the patient from a holistic point of view and to act accordingly.

Keywords: Diabetes Mellitus Type 1, pediatric nursing, adolescent behavior, psychosocial Impact.

\section{RESUMEN}

Durante la adolescencia se presentan multitud de factores psicosociales que pueden alterar el proceso de desarrollo hacia la edad adulta. En adolescentes que además padecen Diabetes Mellitus tipo I, este desarrollo se complica muchísimo más, provocando afectación al mantenimiento adecuado de esta enfermedad crónica.

Objetivo: Conocer la influencia de los factores psicosociales sobre el control de la Diabetes Mellitus en la adolescencia.

Metodología: Se realiza una revisión bibliográfica en las bases de datos PubMed, CINAHL, Scielo, CUIDEN, Índice Médico Español, Dialnet, además de Google Académico y otras web de organizaciones especializadas, seleccionando los artículos de investigación de los últimos 10 años como criterio de inclusión principal.

Resultados-Discusión: Como resultados encontramos como resultados ocho factores principales que influyen en el control de la Diabetes en adolescentes, que son la familia, los amigos, la ansiedad y la depresión, la dieta y alimentación, el estrés, el autoestima, el desarrollo cognitivo y la cultura y religiosidad.

Conclusión: Obtenemos que se deben tener en cuenta, no sólo los factores físicos y biológicos, sino todos los aspectos psicológicos y sociales que también rodean al adolescente. El enfermero debe tener presentes estos factores al tratar con adolescentes, ya que pueden ser éstos los causantes de su desajuste de glucosa en sangre, por lo que siempre es necesario valorar al paciente desde un punto de vista holístico e intervenir en consonancia.

Palabras Clave: Diabetes Mellitus Tipo 1, enfermería pediátrica, conducta del Adolescente, Impacto Psicosocial.

\section{RESUMO}

Durante a adolescência muitos fatores psicossociais que podem alterar o processo de desenvolvimento até a idade adulta são apresentados. Em adição adolescentes com diabetes mellitus tipo I, este desenvolvimento é muito mais complicado, causando comprometimento da manutenção adequada dessa doença crônica.

Objetivos: Conhecer a influência de fatores psicossociais no controle do Diabetes Mellitus na adolescência.

Metodologia: Uma revisão da literatura foi realizada no PubMed, CINAHL, Scielo, CUIDEN, Índice de Medicina espanhol, Dialnet, além de dados do Google Acadêmico e outras organizações especializadas website, selecionando artigos dos últimos 10 anos de investigação como a inclusão primário .

Resultados-Discussão: Como resultados são oito principais fatores que influenciam o controle do diabetes em adolescentes, eles são família, amigos, ansiedade e depressão, dieta e nutrição, stress, auto-estima, o desenvolvimento cognitivo e cultura e religião .

Conclusão: Em conclusão obtemos que deve ser levado em conta não apenas os fatores físicos e biológicos, mas todos os aspectos psicológicos e sociais que também envolvem o adolescente. Enfermeiros devem estar cientes desses fatores quando se trata de adolescentes, uma vez que podem ser a causa de seu dese- 
quilíbrio de glicose no sangue, por isso é sempre necessário avaliar o paciente de um ponto de vista holístico e agir em conformidade.

Palavras chave: Diabetes Mellitus dependente de insulina, adolescencia, aspectos psicossociais, enfermagem pediatrica.

\section{INTRODUCCIÓN}

La Diabetes Mellitus (DM) Tipo I es una de las enfermedades crónicas más prevalentes en España (entre el 0.08 y el 0,2\% en España), siendo una de las enfermedades que más ha evolucionado de manera ascendente en los últimos años a grandes dimensiones. A nivel mundial, casi 400 millones de personas tienen diabetes y se estima que para 2035, esta cifra haya aumentado hasta los 592 millones (Fundación para la Diabetes, 2015; Instituto Nacional de Estadística, 2015).

Se puede definir la DM tal y como la describe la Organización Mundial de la Salud, como "una enfermedad crónica que aparece cuando el páncreas no produce insulina suficiente o cuando el organismo no utiliza eficazmente la insulina que produce " (2015).

Clásicamente, la DM tipo 1 se ha identificado como una diabetes que se presenta en pacientes jóvenes y delgados que presentan dependencia insulínica externa desde sus inicios, ya sea la infancia o adolescencia, dependiendo del momento de su manifestación (Beato, 2012). El diagnóstico de la DM es bastante sencillo, ya que la sintomatología es bastante clara. Aún así, existen una serie de características clínicas que son criterios diagnósticos de la DM tipo I:

- HbA1c mayor o igual a 6,5\%.

- Glucemia en ayunas mayor o igual a $126 \mathrm{mg} / \mathrm{dl}$.
- Glucemia a las 2 horas, tras una Sobrecarga Oral de Glucosa de 75 gramos, mayor o igual a $200 \mathrm{mg} / \mathrm{dl}$.

- Glucemia mayor o igual a $200 \mathrm{mg} / \mathrm{dl}$ en cualquier momento del día.

Por tanto, debemos saber que es necesario el diagnóstico asegurado de esta enfermedad en los pacientes, además de tener en cuenta otras características, como son la actitud terapéutica, el impacto sobre el estilo de vida y la búsqueda de complicaciones, ya que estos son importantes tenerlos en cuenta en relación con la enfermedad (Rubio y Argente, 2012).

Esta enfermedad está presente en diferentes edades, aunque su presencia es mayor en edades adolescentes, siendo el mayor pico de incidencia los 14 años (Beato, 2012; Rubio y Argente, 2012). La adolescencia es una de las etapas más importantes, en la que se originan cambios que pueden reorientar el sentido de la vida para el propio adolescente, estando muy presentes los factores psicosociales que influyen en la enfermedad de la DM. Los factores psicosociales son aquellos componentes que intervienen en el desarrollo de la persona a nivel cognitivo, mental y de interacción con su entorno, entendiéndose este desarrollo como un proceso de transformación, donde permanece de manera constante una interacción de la persona con el ambiente a nivel psicológico y social (Baeriswyl y Montt, 2007).

En este sentido, hay un gran número de personas que deben modificar muchas de las actividades de la vida diaria para poder adaptarlas a las condiciones de la enfermedad, sobre todo adolescentes que se encuentran en un proceso de desarrollo personal en el que coexisten numerosas influencias sociales, psicológicas y emocionales (Luna y García y Barragán, 2014; Rondón, 2011; Silva-Escorcia 
y Mejía-Pérez, 2015). Existen innumerables investigaciones y artículos que desarrollan cada una de las múltiples ayudas biomédicas que favorecen la regulación biológica del organismo, incidiendo en los factores físicos alterados por los que se caracteriza la DM tipo I (Beato, 2012; Alves, Lottemberg, Lottemberg, Della Manna y Kuperman, 2009; Miranda, Domínguez, Arroyo, Méndez y González, 2012). Sin embargo, las Guías de Práctica Clínica más conocidas no aportan información sobre los aspectos psicosociales que influyen en el control de la diabetes, lo hacen de manera superficial o incluso presentan controversias al respecto, especialmente para la población adolescente (American Diabetes Association, 2015; Andrade, Vargas, do Nascimiento, das Neves y Galvão, 2011; Federación de Diabéticos Españoles, 2015; Fundación para la Diabetes, 2015; Guía de Práctica Clínica sobre Diabetes Mellitus Tipo I, 2015; Revista Diabetes, 2014). Dada la trascendencia de estos factores para el manejo de la diabetes por los adolescentes, resulta necesario identificar dichos factores en la literatura y el papel de la enfermera al respecto.

\section{METODOLOGÍA}

Para dar respuesta a esta situación, se ha realizado una revisión crítica y reflexiva de la literatura en las principales base de datos y hemerotecas anglosajonas e iberoamericanas, tales como PubMed, CINAHL, Scielo, CUIDEN, Índice Médico Español, Dialnet, además de Google Académico y otras web de organizaciones especializadas.

Para seleccionar los artículos, debían cumplir una serie de criterios, tales como haber sido publicado en los últimos 10 años y ser artículos de investigación que traten la influencia de los factores psicosociales que rodean a los adolescentes que padecen DM tipo I en el óptimo control glucémico de la enfermedad, descartándose aquellos que no tuvieran relación en su resumen y desarrollo con nuestro tema a revisar.

\section{RESULTADOS-DISCUSIÓN}

Se han encontrado diversos factores psicosociales que pueden influir en el control de la DM y que pueden agruparse en ocho categorías. Además, se presenta el papel de las enfermeras en relación a dichos factores. Así, se presentan:

\section{Familia}

La presencia de una enfermedad crónica en algún miembro de una familia acarrea múltiples condicionantes que pueden implicar daños a nivel afectivo en el joven. Los resultados de un estudio realizado por Teixeira Leal D (2012) corroboran la influencia de este factor en el control glucémico del paciente. En él, se reflejan las experiencias negativas (estrés, negativismo, cambios repentinos en el estado de ánimo...) de muchos familiares al tener que cambiar diversas actividades cotidianas para adaptarlas a la enfermedad crónica de su familiar. El hecho de no tener este sustento familiar adecuadamente puede conllevar a la desorganización de aspectos psicológicos como la alteración de la dieta que debe seguir o la presencia de estrés (Moore, Hamilton, Northam y Cameron, 2013; Texeira, Andrade, Vargas, do Nascimento y Cristiane, 2012).

Según el estudio realizado por Martínez B (2007), tan sólo la mitad de los padres de hijos diabéticos reconocían ser conscientes de las inquietudes y dudas de su hijo y su relación con su enfermedad. Esto provoca más malestar en el paciente, llevando la situación a un descontrol de los cuidados necesarios y, por tanto, al descontrol glucémico que impli- 


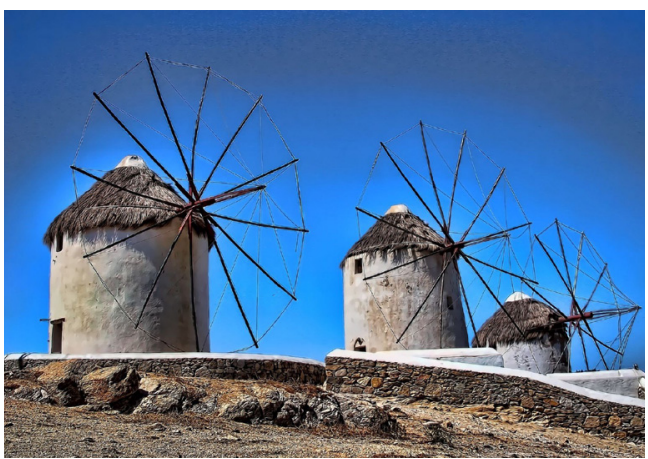

ca esta enfermedad. Este estudio revela que un $92 \%$ del grupo estudiado reconocía que la responsabilidad de la medicación recae sobre la madre, lo que implica un descontrol del tratamiento si se tiene malas relaciones con el progenitor. Un tercer estudio realizado nos muestra que muchos de los padres y adolescentes ven modificada su vida en relación a la aparición de la enfermedad en el joven (Jaser et al, 2012; Martínez y Torres, 2007).

\section{Amistad}

Para todo adolescente, pertenecer a un grupo social y estar integrado en él, implica el propio bienestar psicológico; llevando consigo la aceptación de uno mismo y el fortalecimiento del autoestima (Silva-Escorcia y Mejía-Pérez, 2015).

En un estudio cualitativo realizado por Cássia Sparapani V (2012), se demuestra cómo influye positivamente el apoyo y el cariño de los amigos para el afrontamiento de la enfermedad por parte del adolescente enfermo. La amistad más próxima se muestra esencial para estos pacientes, pero no sólo en los momentos más complicados, sino en las dificultades del día a día. También se hace referencia a la dificultad que tienen estos jóvenes con DM para la integración, sometidos con frecuencia a acoso escolar por su particular estilo de vida (Sparpanni, Borges, Dantas y Nascimento, 2012).

\section{Ansiedad y depresión}

En un estudio de corte empírico-analítico realizado por Moreno Méndez (2012) en la Unidad de Servicios Psicológicos de la Universidad Católica de Colombia, demuestra que la mayoría de los pacientes ingresados se encontraban en la etapa adolescente y los problemas psicológicos más frecuentes fueron, entre otros, la ansiedad y la depresión (Moreno, Rozo, Cantor y Toro, 2012).

Si con esto datos, podemos pensar que la adolescencia es una etapa propensa a desarrollar problemas psicológicos, una revisión bibliográfica realizada por Souza Duarte $\mathrm{N}$ (2012) nos muestra la gran relación que existe entre la presencia de esta enfermedad crónica y, a su vez, problemas en el funcionamiento neuropsicológico de la persona (Souza, Oliveira, Lima, Hazín y Arrais,2012).

Un estudio realizado por Hughes (2011) sobre la relación del estado anímico con la cantidad de HbAlc muestra la estrecha relación de estas afectaciones psicológicas con el adecuado mantenimiento metabólico que es alterado por la enfermedad (Souza et al, 2012; Hugues, Berg y Wiebe, 2012).

\section{Alimentación}

El hecho de padecer DM en la adolescencia hace más propensa la aparición de trastornos de la alimentación que ya son muy frecuentes entre los jóvenes. Se provoca pues una especie de "círculo vicioso" en la que la alimentación se ve afectada por estos factores $y$, a su vez, la enfermedad se ve alterada por la falta de alimentación equilibrada, algo imprescindible para esta enfermedad crónica (Alves, Pinto, Bastos y Carcalheiro, 2011; Souza et al,2012).

Un estudio transversal basado en un periodo de 2008 a 2010 demostró que, en la población estudiada, aquellos que presentaban 
la $\mathrm{HbAl}$ alterada tenían más dificultad para la adherencia a la dieta diabética, siendo las principales quejas la cantidad de alimento y el horario de las comidas. Sin embargo, aquellos que confirmaban tener una buena adherencia a la dieta presentaba valores adecuados de Hb1A (Davison et al, 2014).

\section{Estrés}

El estrés de los adolescentes está estrechamente relacionado con la enfermedad. El hecho de ser conscientes de tener que controlar muchas actividades cotidianas se vive de antemano como una experiencia negativa, que provoca en muchos jóvenes la aparición de estrés. La adherencia terapéutica, por ejemplo, es una conducta que acarrea un efecto estresante $y$, además, la propia enfermedad de la diabetes crea un efecto sobre el metabolismo del paciente adolescente que favorece el estrés (Rondón, 2011; Tran, Wiebe, Fortenberry, Butler y Berg, 2011).

En el estudio realizado por Tran V (2011) se refleja la relación de las variables que los propios adolescentes del estudio perciben como estresantes y su percepción sobre el manejo de la enfermedad relacionado con el buen control y mantenimiento de la enfermedad a nivel metabólico. Aquellos adolescentes con reacciones depresivas estaban asociadas a estrés por la enfermedad, y la aparición de depresión y, como consecuencia, de estrés, tenían estrecha relación con la HbAlc más baja.

La presencia de estrés en este tipo de personas estimula también el desorden del autocontrol $\mathrm{y}$, por tanto, también de las conductas saludables que se deben llevar a cabo (Tran et al, 2011).

\section{Autoestima}

La autoestima es un factor muy importante en los adolescentes tanto para ellos mismos como para su relación con el medio que le rodea, bien a nivel educativo o social, y también para el proceso de control de su DM tipo I. Muchos de los adolescentes tras ser diagnosticados de esta enfermedad crónica deterioran la imagen de sí mismo, considerándose como personas enfermas que no tienen el control de sus vidas, sino que están crónicamente condicionadas por las actividades que les rige la enfermedad (Baeriswyl et al, 2007; Silva-Escorcia y Mejía-Pérez, 2015).

José Eduardo Rondón Bernard reflexiona en su trabajo si la diabetes modifica la autoestima de los adolescentes o son sus complicaciones las que alteran esa autoestima. De una manera u otra, los adolescentes con DM son propensos a experimentar estos cambios sobre su autoconcepto. Tal es así que puede llegar incluso a alterar y a descontrolar los niveles de glucosa en sangre (Rondón, 2011).

\section{Desarrollo cognitivo}

Existen estudios en los que se habla de cómo afecta el desarrollo cognitivo del adolescente en el mantenimiento de su enfermedad crónica. Sin embargo, no se describe tanto como factor sino como consecuencia de otros factores. En el caso de José Eduardo Rondón en su estudio de 2011, habla de las alteraciones en la cognición como consecuencia de una baja autoestima y, por tanto, también del negativismo del joven.

Todo ello en su conjunto provoca el rechazo a la adecuación de su vida diaria para un buen control de la DM (Rondón, 2011). Precisamente, en el trabajo de Silva-Escorcia (2015) se recalca aún más esta relación autoestimanivel cognitivo. Los resultados académicos se ven estrechamente influidos por esta relación, teniendo en cuenta que aquello que disminuye la autoestima del joven es el padecimiento de 
la DM Tipo I (Silva-Escorcia y Mejía-Pérez, 2015).

\section{Cultura y religión}

Es posible encontrar en diversas publicaciones la relación del buen mantenimiento de la enfermedad crónica con la práctica de una religión u otra, o el hecho de pertenecer a una cultura con determinadas características. Se une a estas circunstancias, el aumento de adolescentes de distintas culturas que los enfermeros deben cuidar, por lo que la trascendencia de este factor es cada vez mayor si se tiene en cuenta este aspecto transcultural tanto en los adolescentes como en los profesionales. Partiendo de la base de la que surge la reflexión de José Eduardo Rondón Bernard, muchas de nuestras actividades de la vida diaria están condicionadas por los factores culturales con los que hemos crecido inconscientemente y algún determinado estilo de vida puede provocar riesgos en nuestra salud. Desde este punto de vista, podemos encontrar adolescentes que incumplen por razones culturales algunas de las actividades necesarias para mantener la $\mathrm{DM}$ en sus adecuados niveles. Es un factor a tener en cuenta si esto es lo que principalmente altera el mantenimiento de la enfermedad en algún joven en esta situación (Rondón, 2011; Castrillón, 2015).

\section{Enfermería y adolescentes con dm tipo $i$}

La influencia del trabajo de los enfermeros sobre estos pacientes es de vital importancia, ya que de ella depende, en gran parte, que el mantenimiento de los cuidados para controlar la DM se mantenga correctamente (Bareiswyl, 2007; Carvalhêdo, Moura, Girão, Freire y Coelho, 2010). De esta manera, el enfermero debe ser capaz de comprender la situación de este paciente tan complejo teniendo en cuenta las características de la persona holísticamente e incorporar los cuidados necesarios para la DM adaptándolo a la vida diaria de manera individual (Rondón, 2011), desde un modelo enfermero que permita un abordaje transcultural, imprescindible en la sociedad actual (Lopez Alonso SR, 2003).

Claramente se demuestra la importancia de los enfermeros en la vida de estos pacientes en el estudio cualitativo realizado en 2010 por Fragoso LVC et al, donde se demuestra la percepción de los adolescentes del papel de los enfermeros, ya que su profesionalidad disminuye la ansiedad y la depresión en ellos, gracias al apoyo emocional, el afecto demostrado $y$, en general, el buen manejo de las necesidades, tanto a nivel físico o biológico como a nivel emocional. Todo ello conlleva a una mejora de las actividades de la vida diaria, a una mejor adhesión al tratamiento y a un bienestar psicológico que contribuye, en general a una mejora de la calidad de vida (Carvalhêdo, 2010).

\section{CONCLUSIÓN}

Existen determinados factores que afectan al control de la Diabetes Mellitus tipo I en la adolescencia. Las consecuencias de la alteración de estos factores son muy variadas. Por un lado, su desajuste provoca consecuencias tales como falta de adherencia al tratamiento, descuidos en las actividades diarias o negación a los cuidados especiales, provocando a su vez la aparición de hipoglucemias o hiperglucemias, cetoacidosis, etc. Por otro lado, el positivismo ante la situación, la adherencia al tratamiento y la preocupación por realizar correctamente las actividades de la vida diaria conlleva a buenos niveles de glucosa en sangre y buena calidad de vida en general.

Gracias a las actuaciones de los enfermeros con los adolescentes, se facilita a estos pacien- 
tes llevar un mejor control de su enfermedad. Actuar conjuntamente el enfermero y el adolescente ayuda a superar el problema en sí que dificulta el día a día del joven y hacer mucho más llevaderas las actividades diarias necesarias para su óptimo control.

\section{REFERÊNCIAS}

- Alves, S.A., Lottenberg, S.A., Lottenberg, A.M., Della Manna, T., \& Kuperman, H. (2009). Home blood glucose monitoring in type 1 Diabetes Mellitus. Rev Latino-am Enfermagem, 17(2), 194-200.

- Alves, M., Rodrigues, D., Pinto, J., Bastos, M., \& Carvalheiro, M. (2011). Doenças do comportamento Alimentar e diabetes mellitus Tipo 1. Acta Med Port, 24(3),639-646.

- American Diabetes Association [actualizado 1 Enero 201; citado 10 Febrero 2015]. Recuperado de http://www. diabetes.org/es/

- Andrade, F., Vargas, I.M.A., do Nascimiento, L., das Neves, P., \& Galvão, S. (2011). Crianças e Adolescentes com Diabetes Mellitus: Cuidados/Implicações Para a Enfermagem. Rev. Latino-am Enfermagem, 25(2),145-154.

- Baeriswyl, K. Montt, M.E. (2007). Salud mental, factores psicológicos y familiares en niños diabéticos tipo 1 entre los 8 y los 12 años que viven en la V Región. Santiago: Universidad de Chile.

- Beato, P.I. (2012). Características clínicas, inmunológicas y genéticas en el debut de Diabetes Mellitus tipo 1 en adolescentes y adultos y su influencia en el control metabólico y la variabilidad glucémica posteriores. Cáceres: Universidad de Extremadura.

- Bjarkøy, R., Graue, M., Wentzel-Larsen,T., Peyrot, \& M, Rokne. B . (2014). Relationships of diabetes-specific emotional distress, depression, anxiety and overall well-being with $\mathrm{HbA} 1 \mathrm{c}$ in adult persons with type 1 diabetes. Journal of Psychosomatic Research, 77,174-179.

- Carvalhêdo, L., Moura, M., Girão, A., Freire, R., \& Coelho, M. (2010). Vivências Cotidianas De Adolescentes Com Diabetes Mellitus Tipo 1. Rev. Latino-am Enfermagem,19(3):443-451.

- Castrilló,, E.(2015). La enfermera transcultural y el de- sarrollo de la competencia cultural. Cultura de los Cuidados, 42,128-136.

- Davison K., Negrato, C., Cobas, R., Matheus, A., Tannus, L., Palma, C.S., et al. (2014). Relationship between adherence to diet, glycemic control and cardiovascular risk factors in patients with type 1 diabetes: a nationwide survey in Brazil. Nutrition Journal, 13(19),1-11.

- Fundación para la Diabetes. “Comenzar bien el día”. Día mundial de la diabetes. [Consultado el 17.02.2015], de Fundación para la Diabetes. Recuperado de: http://www. fundaciondiabetes.org/upload/publicaciones_ficheros/68/guiapractica_dmd2014.pdf

- Fundación para la Diabetes. Se puede vivir con Diabetes. Juventud y Diabetes: Breve guía para entender y controlar la diabetes tipo 1 Consultado el 11.03.2015], de Fundación para la Diabetes. Recuperado de http://www.fundaciondiabetes.org/upload/publicaciones_ficheros/54/ Diptico.pdf

- Federación de Diabéticos Españoles. Me acaban de diagnosticar Diabetes Mellitus Tipo 1 (2012). [Consultado el 10.03.2015], de Federación de Diabéticos Españoles. Recuperado de: http://docsafedownload.net/fedesp/ bddocs/1/ME-ACABAN-DE-DIAGNOSTICAR-QUE-TENGO-DIABETES-TIPO-1.pdf

- Hughes, A.E., Berg,C.A., \& Wiebe, D.J (2012). Emotional Processing and Self-Control in Adolescents With Type 1 Diabetes. Journal of Pediatric Psychology, 37(8),925-934.

- Instituto Nacional de Estadística [actualizado 3 Febrero 2015; citado 10 Febrero 2015]. Recuperado de http:// www.ine.es/

- Jaser, S.S., Faulhner, M., Whittemore, R., Jeon, S., Murphy, K., Delamater, A., et al. (2012). Coping, Self-Management, and Adaptation in Adolescents with Type 1 Diabetes. Ann Behav. Med, 43(3),311-319.

- López Alonso, S.R. (2003). Afrontamiento enfermero ante la desigualdad y el racismo. Cultura de los Cuidados, 13:71-77.

- Luna Rodríguez, A.G., \& García y Barragán, L.F. (2014). El modelo transteórico y adherencia terapéutica en adolescentes con Diabetes Mellitus tipo I. Revista Ibero Americana de Psicología,7(1),49-58.

- Miranda Velasco, M.J., Dominguez Martín, E., Arroyo 
Díez, F., Méndez Pérez, P., \& González de Buitrago Amigo, J. (2012). Calidad de vida relacionada con la salud en la diabetes mellitus tipo 1. An pedriatr, 77(5), 329-333.

- Ministerio de Sanidad, Servicios Sociales e Igualdad. Guía de Práctica Clínica sobre Diabetes Mellitus Tipo 1. 2012. [Consultado el 02.01.2015]. Recuperado de http:// www.guiasalud.es/GPC/GPC_513_Diabetes_1_Osteba_compl.pdf

- Martínez Martínez, B., \& Torres Velázquez, L.E. (2007). Importancia de la familia en el paciente con diabetes mellitus insulinodependiente. Psicología y Salud, 17(2), 229-240.

- Moore, S.M., Hackworth, N.J., Hamiltomn, V.E., Northam, E.P., \& Cameron, F.J. (2013). Adolescents with Type 1 Diabetes: parental perceptions of child health and family functioning and their relationship to adolescent metabolic control. Health and Quality of Life Outcomes, 11(50),1-8.

- Moreno Méndez, J.H. Rozo Sánchez, M.M., Cantor Nieto, M.I., \& Toro Tobar, R.A (2012). Características psicosociales de la población asistente a la unidad de servicios psicológicos de la universidad católica de Colombia durante los años 2007 y 2008. Psychol. av. discip, 6(1),21-33.

- Organización Mundial de la Salud [actualizado 1 Abril 2015; citado 10 Febrero 2015]. Recuperado de http:// www.who.int/es/

- Rubio Cabezas, O., \& Argente, J. (2012). Diabetes Melli- tus: formas de presentación clínica y diagnóstico diferencial de la hiperglucemia en la infancia y adolescencia. An pediatr, 77(5),344.e1-344.e6.

- Rondón Bernard, J.E. (2011). Variables psicosociales implicadas en el mantenimiento y control de la Diabetes Mellitus: aspectos conceptuales, investigaciones y hallazgos. Revista Electrónica de Psicología Iztacala, 14(2),126162.

- Ros Pérez, P. ¿Es preferible que los niños estén un poco altos de glucosa?. [Consultado el 13.12.2014] Recuperado de http://www.revistadiabetes.org/media/revista/31/ la\%20consulta.pdf

- Silva-Escorcia, I.,\& Mejía-Pérez, O. (2015). Autoestima, adolescencia y pedagogía. Revista Electrónica Educare, 19(1),241-256.

- Sparapani, V.de C., Borges, A.L.V., Dantas, I.R.de.O., \& Nascimiento, L.C. (2012) Children with Type 1 Diabetes Mellitus and their friends: the influence of this interaction in the management of the disease. Rev. Enferm, 20(1),1-9. - Souza Duarte, N., Oliveira Carcalho, M.F., Lima Freire, R.C, Hazín, I.,\& Arrais, R.A. (2012). Type 1 Diabetes Mellitus and Neuropsychology Abilities: Literature Review. Rev. Latino-am Enfermagem, 6(12),3032-3040.

- Tran, V., Wiebe, D.J., Fortenberry, K.T. Butler, J.M., \& Berg, C.A. (2011). Benefit Finding, Affective Reactions to Diabetes Stress, and Diabetes Management among Early Adolescents. National Institute of Health, 30(2),212-219. 